\title{
Reactive Oxygen Species Over Production and Superoxide Dismutase Exhaustion Promoting the Intervertebral Disc Degeneration
}

\author{
Cencen Hong ${ }^{1}$ and Lin Ouyang ${ }^{2 *}$ \\ ${ }^{1}$ Graduate School of Medical, China \\ ${ }^{2}$ Department of Medical Imaging, China \\ *Corresponding author: Lin Ouyang, Department of Medical Imaging, Zhangzhou, China
}

ARTICLE INFO

Received: 幽 March 08, 2019

Published: 慧 March 19, 2019

Citation: Cencen Hong, Lin Ouyang. Reactive Oxygen Species Over Production and Superoxide Dismutase Exhaustion Promoting the Intervertebral Disc Degeneration. Biomed J Sci \& Tech Res 16(1)-2019. BJSTR. MS.ID.002801.

Keywords: Intervertebral Disc Degeneration; Oxidative Stress; Reactive Oxygen Species; Superoxide Dismutase

\begin{abstract}
Background: Oxidative overstress is realized as one of the most prevalent causes of Intervertebral Disc Degeneration (IVDD) by restraining the metabolism and damaging the cell matrix, but the specific process is not yet well known. To explore the specific relationship of Reactive Oxygen Species (ROS) and Superoxide Dismutase (SOD) with IVDD.

Methods: ICR mice were managed with progressive loading of Upstanding Time (UST) to prepare serial levels of IVDD mouse models. ROS and SOD, as the Oxidative Stress (OS) indexes of the endplate and Intervertebral Disc (IVD) from upright mechanical stress, were longitudinally assessed by immunohistochemistry. The endplate cells and structures were synchronously dynamically assayed by histopathology.

Results: SOD content in the complexs of the endplate and IVD rapidly increased within 1 to 3 days in the earlier stage after upstanding mechanical stress experiment. Conversely, the ROS level was inhibited until the day 3 after upstanding. Then, SOD decreased gradually, and ROS increased reversely with UST lasting. The histopathology showed that the endplate cartilage cells well-arranged and homogeneous with clear endplate boundaries in the control and 1-day upstanding groups, and the endplate cells swollen until the day 3 after upstanding. With UST lasting, the endplate cells became lysis and sparse, and various structures showed disruption, collapse and blurred in the 9-day group.
\end{abstract}

Conclusion: Oxidative stress is closely related with IVDD, ROS over production and SOD compensatory depletion activating the IVDD.

\section{Introduction}

Human Intervertebral Discs (IVD) may undergo a series of biochemical and then morphological changes as early as the second decade of life [1]. These degenerative changes can be induced by lumbar pressure changes, spinal instability, annular injury, chemical damage or gene deficiency, and usually deteriorate further with aging [2-4]. Overstress is realized as one of the most prevalent causes of Intervertebral Disc Degeneration (IVDD) by restraining the metabolism and damaging the cell matrix [5]. Nevertheless, both short and prolonged mild over stresses are often ignored as having an effect on the uniform stress distribution on the IVDs [6].
Early prediction can provide opportunities for disease progression interventions that may ameliorate indications rather than treating late-stage symptoms. Thus, early identification of the respective biomarkers during the progress of IVDD is necessary. A series of subtle metabolic disorders have been reported to take place in the IVD internal environment, including blood vessels reduction, autoimmune inflammation, enzyme inactivity and apoptosis [7]. These response activities lead to the chronic inflammation featured by the production and accumulation of Reactive Oxygen Species (ROS) that may take place long before any morphology changes [2,3]. Oxidative Stress (OS) 
is further intensified by the overproduction of ROS due to abnormal cell metabolism that exceeds the physiological buffering capacity of cells, ultimately resulting in damaged carbohydrates, lipids, proteins, nucleic acids, amino acids, vitamins and matrix components [5].

The endogenous excessive ROS production, antioxidant defense homeostasis or impaired antioxidant Defense mechanisms during inflammation caused by stress-induced IVDD generally determine the degree of OS on the vertebral body (i.e. centrum) [8]. Superoxide Dismutase (SOD) plays an important role in protecting the basic cell function and reduces tissue damage [9]. Taken in concert, these key factors, OS, ROS and SOD, during an IVDD progression are equally examined to identify the role of each biomarkers in this study. For the purpose, animal models were studied longitudinally in vitro through histopathology and immunobiology.

\section{Materials and Methods}

\section{IVDD Animal Models}

All procedures were approved by the Institutional Animal Care and Use Committee of Xiamen University. ICR mice (male, 5 weeks of age, 22-25 g) were obtained from the Animal Center of Xiamen University. All animals were individually housed and fed with standard rat pellets. The mice were divided into five groups (12 randomly selected animals each group) and labelled with the corresponding group label (A, B, C, D and E). Each mouse in group A was put in a slender PVC pipe (internal diameter $35 \mathrm{~mm}$, height $150 \mathrm{~mm}$ ) to enable the mouse to stand on both front feet 8 hours per day for 1 day [10]. The mice in groups B, C, D and E were placed in the same pipes 8 hours per day for 3, 5, 7 and 9 days, respectively. Net lids were used to cover the pipes. When the mice were forced to stand or squat on their hind feet in the pipe, their whole-body weight was supported by the lumbar and the strain caused IVDD, mimicking the lumbar spine injury in humans during prolonged standing and sitting (Figure 1). High-field MR scanning was performed and analyzed for each group to verify the severity of IVDD. IVD and centrums were collected for obtaining the Nucleus Pulposus (NP) tissues and endplates immediately after all in vivo experiments and homogenized for oxidative stress analysis. Tissues from the IVD and centrums of L4 and L5 were subsequently harvested for histomorphometry, cell morphology and immunohistochemistry [6].

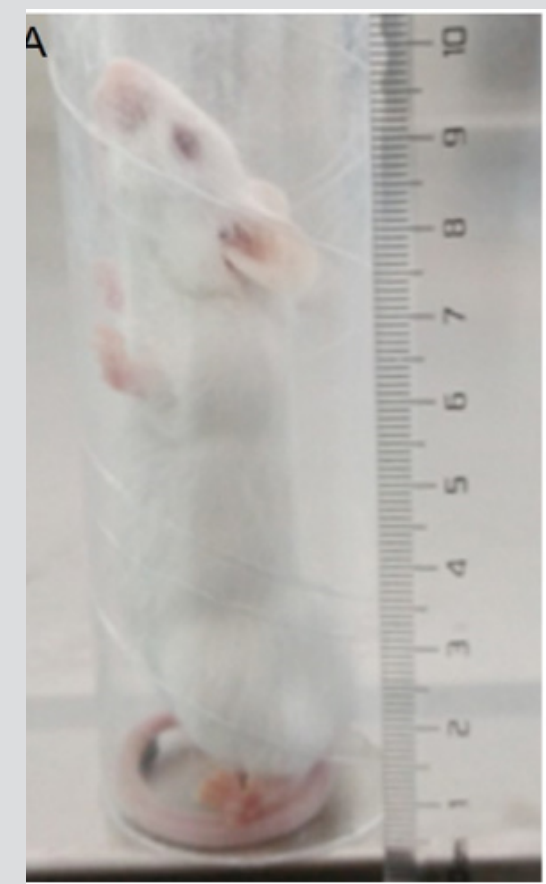

Figure 1: The IVDD mice model establishment.

\section{Histology Analysis}

To validate the IVDD morphology changes and level decreases in collagen type II that mainly exists in the NP during lumbar disc degeneration [11], tissues from the endplate and IVD (L4, L5) of each group were obtained for histologic and cytology analysis. The tissues were decalcified en bloc using a JYBL-IIdecalcifying solution (Co., Yumu., Shanghai agent,

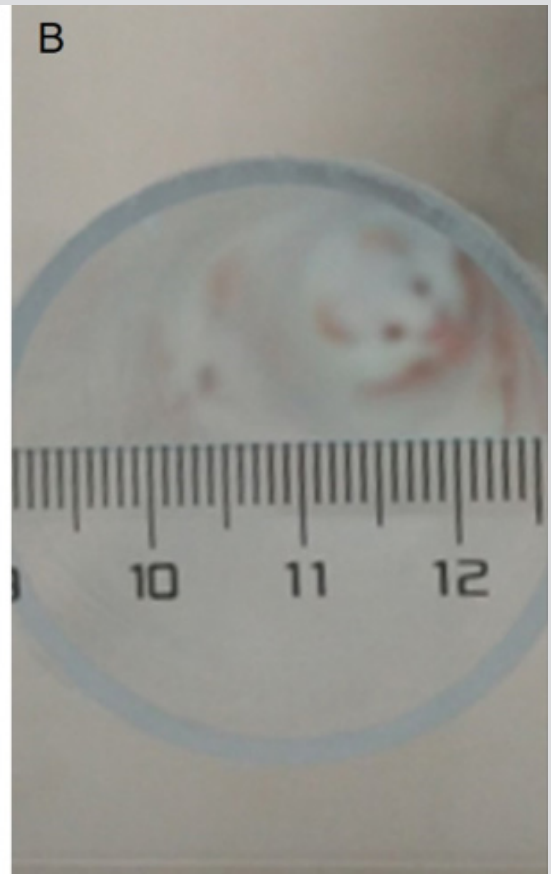

China) before the centers of the vertebrae were cut off. Then, the tissues were fixed in 10\% neutral buffered formalin and embedded in paraffin. The paraffin blocks were cut into $3-\mu \mathrm{m}$ thick sections, which were then dewaxed with xylene and hydrated with graded ethanol. Subsequently, the sections were stained with Safranin O staining solution and sirius red staining solution for histological examination [12]. The 
endplate and IVD structure were analyzed on stained images of the histological sections by using photonic microscopy (Nikon France S.A, Champignysur Marne, France).

Enzyme-Linked Immunosorbent Assay (ELISA). To determine the relationship of IVDD with ROS and SOD production in the IVDD progression, the SOD content and ROS level in the IVD and endplate tissues were measured by a commercially available ELISA kit (Co., Yumu., Shanghai agent, China) according to the instructions provided by the manufacturer. Briefly, the end plates (L4-L5) were extracted by centrifugation of mice samples. The assay was based on the activity of this enzyme in the enzyme catalysis reaction system and the changes in absorbance were measured at $450 \mathrm{~nm}$ using a UV-spectrophotometer.

\section{Statistical Analysis}

The collected data were presented as mean \pm Standard Deviation (SD). Statistical analysis was performed using a One-Way Variance Test (ANOVA) with GraphPad Prism 6 statistical software, that was used for comparative the differences of all assessed factors between groups in vivo and ex vivo. All experimental road map was shown in Scheme 1.

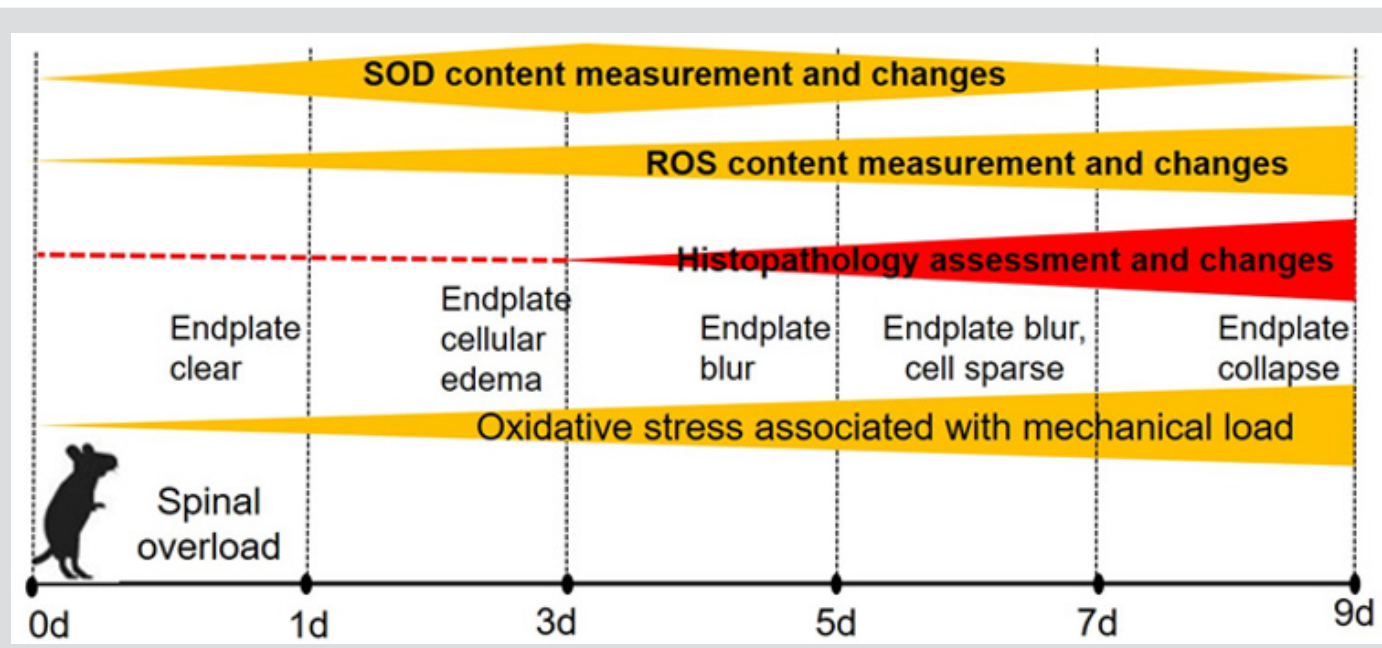

Scheme 1: Experimental road map.

\section{Results}

\section{Oxidative Stress Factors Immunohistochemistry Analysis}

In the study, we found that SOD content of the endplate and IVD rapidly increased within 1 to 3 days in the earlier stage of an upstanding mechanical stress experiment, and then decreased gradually with time of mechanical development of overload-induced IVDD. On the contrary, the ROS levels were inhibited within 1 to 3 days, followed by an increase as the upright experiment being continued. These indicators were quantified as shown in Table 1 and Figure 2.

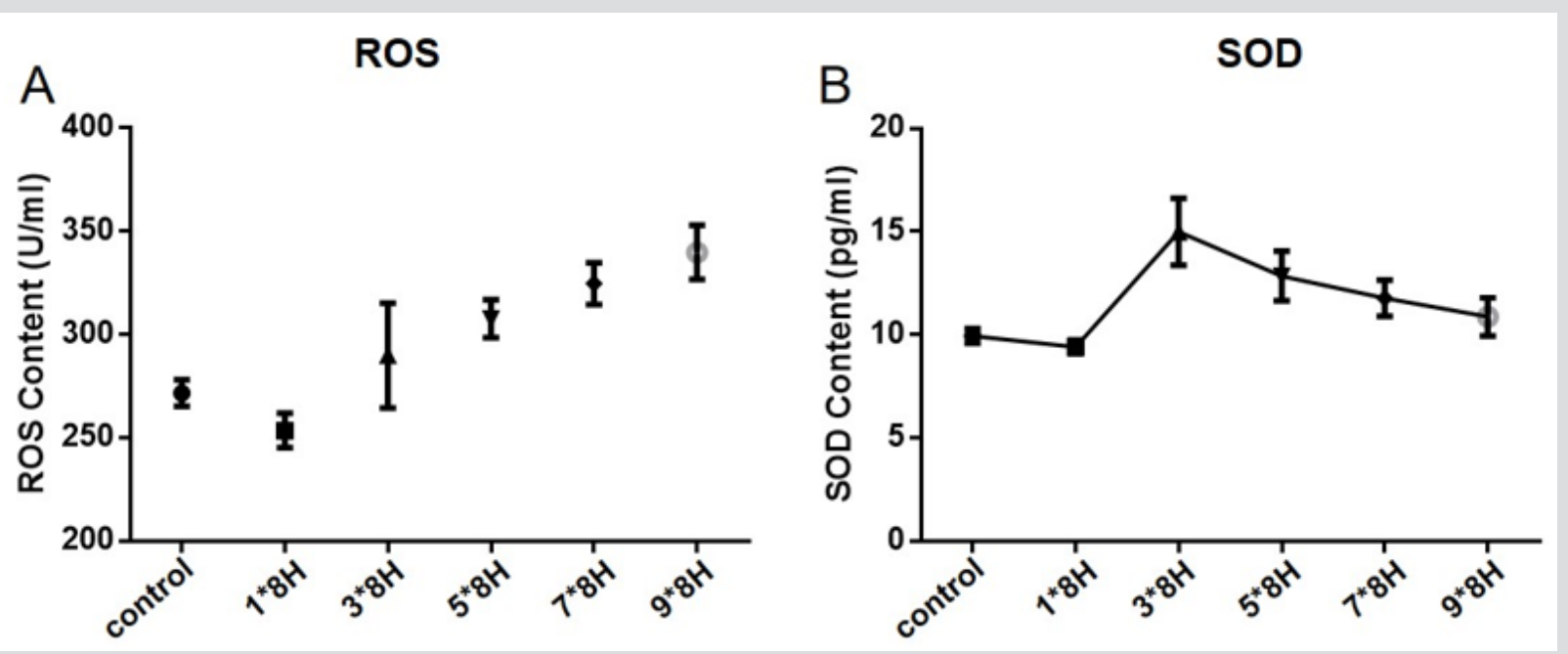

Figure 2: Dynamic changes of SOD and ROS during upright experiment. The SOD content of the IVD rapidly increased to the peak within 3 days and then decreased gradually (A). On the contrary, the ROS levels were inhibited within 1-3 days and followed by increasing (B). This reverse fluctuation revealed the antioxidant activity enhancement of SOD in the early stage of oxygen stress associated with mechanical load and ROS level increasing until compensatory failure of SOD. 


\section{Histopathology Assessment of IVDD}

Safranin and Sirius Red staining were used for the detection of endplate and IVD structure changes. The endplates exhibited obvious changes with time with either of the two staining types. In the control group, cartilage cells were found well arranged, whereas the structure and shape were homogeneous with clear endplate boundaries. However, in the $8 \mathrm{~h} * 3 \mathrm{~d}$ group, the change of endplate could be confirmed by cells swelling on histologic analysis. As time progressed and IVDD became severe, the cells of the endplate and IVD became sparse in number in the $8 h^{*} 5 \mathrm{~d}$ group. And the mouse endplate collapsed in group E $\left(8 h^{*} 9 \mathrm{~d}\right.$ group) (Table 1 and Figure 3).

Table 1: Histopathology of mice at dynamic observation times during upstanding experiment.

\begin{tabular}{|c|c|c|c|c|c|c|c|c|c|c|c|c|c|}
\hline \multirow{2}{*}{\multicolumn{2}{|c|}{ Groups }} & \multirow{2}{*}{\multicolumn{2}{|c|}{$\begin{array}{l}\text { Control group } \\
(n=12)\end{array}$}} & \multicolumn{10}{|c|}{ Treatment groups } \\
\hline & & & & \multicolumn{2}{|c|}{$\begin{array}{l}\text { A group } \\
(n=12)\end{array}$} & \multicolumn{2}{|c|}{$\begin{array}{l}\text { B group } \\
(n=12)\end{array}$} & \multicolumn{2}{|c|}{$C$ group $(n=12)$} & \multicolumn{2}{|c|}{$D$ group $(n=12)$} & \multicolumn{2}{|c|}{ E group $(n=12)$} \\
\hline \multicolumn{2}{|c|}{ Standing duration } & $0 \mathrm{~h}$ & & $1 d^{*} 8 \mathrm{~h}$ & & $3 d * 8 h$ & & $5 d^{*} 8 h$ & & $7 d * 8 h$ & & $9 d^{*} 8 \mathrm{~h}$ & \\
\hline \multicolumn{2}{|c|}{ Tissue harvesting } & Killed & 12 & Killed & 12 & Killed & 12 & Killed & 12 & Killed & 12 & Killed & 12 \\
\hline \multicolumn{2}{|c|}{ Microscope of End plate } & Clear & 12 & Clear & 12 & $\begin{array}{l}\text { Cellular } \\
\text { Edema }\end{array}$ & 12 & $\begin{array}{l}\text { Endplate } \\
\text { blur }\end{array}$ & 11 & $\begin{array}{l}\text { Endplate } \\
\text { blur } \\
\text { and cell } \\
\text { sparse }\end{array}$ & 12 & $\begin{array}{l}\text { Endplate } \\
\text { Collapse }\end{array}$ & 12 \\
\hline \multirow{4}{*}{ Immunohistochemistry } & $\begin{array}{c}\text { SOD } \\
(\mathrm{pg} / \mathrm{ml})\end{array}$ & \multicolumn{2}{|c|}{$9.9 \pm 0.3$} & \multicolumn{2}{|c|}{$9.4 \pm 0.3$} & \multicolumn{2}{|c|}{$15.0 \pm 1.6$} & \multicolumn{2}{|c|}{$12.8 \pm 1.2$} & \multicolumn{2}{|c|}{$11.8 \pm 0.9$} & \multicolumn{2}{|c|}{$10.9 \pm 0.9$} \\
\hline & $\begin{array}{l}\text { One- } \\
\text { way } \\
\text { ANOVA }\end{array}$ & \multicolumn{12}{|c|}{$\begin{array}{c}\mathrm{F}=161.5, \mathrm{p}<0.001 \text { (Differences among means were statistically significant, except control vs. B and control vs. } \mathrm{C} \\
\text { groups) }\end{array}$} \\
\hline & $\begin{array}{c}\text { ROS (U/ } \\
\mathrm{ml})\end{array}$ & \multicolumn{2}{|c|}{$271.6 \pm 10.1$} & \multicolumn{2}{|c|}{$253.4 \pm 13.3$} & \multicolumn{2}{|c|}{$289.8 \pm 39.9$} & \multicolumn{2}{|c|}{$307.7 \pm 14.6$} & \multicolumn{2}{|c|}{$324.7 \pm 15.9$} & \multicolumn{2}{|c|}{$339.8 \pm 20.5$} \\
\hline & $\begin{array}{l}\text { One- } \\
\text { way } \\
\text { ANOVA }\end{array}$ & \multicolumn{12}{|c|}{$\begin{array}{c}\mathrm{F}=27.66, \mathrm{p}<0.001 \text { (Differences among means were statistically significant, except control vs. } \mathrm{B} \text {, control vs. } \mathrm{C} \text { and } \mathrm{C} \\
\text { vs. D groups) }\end{array}$} \\
\hline
\end{tabular}

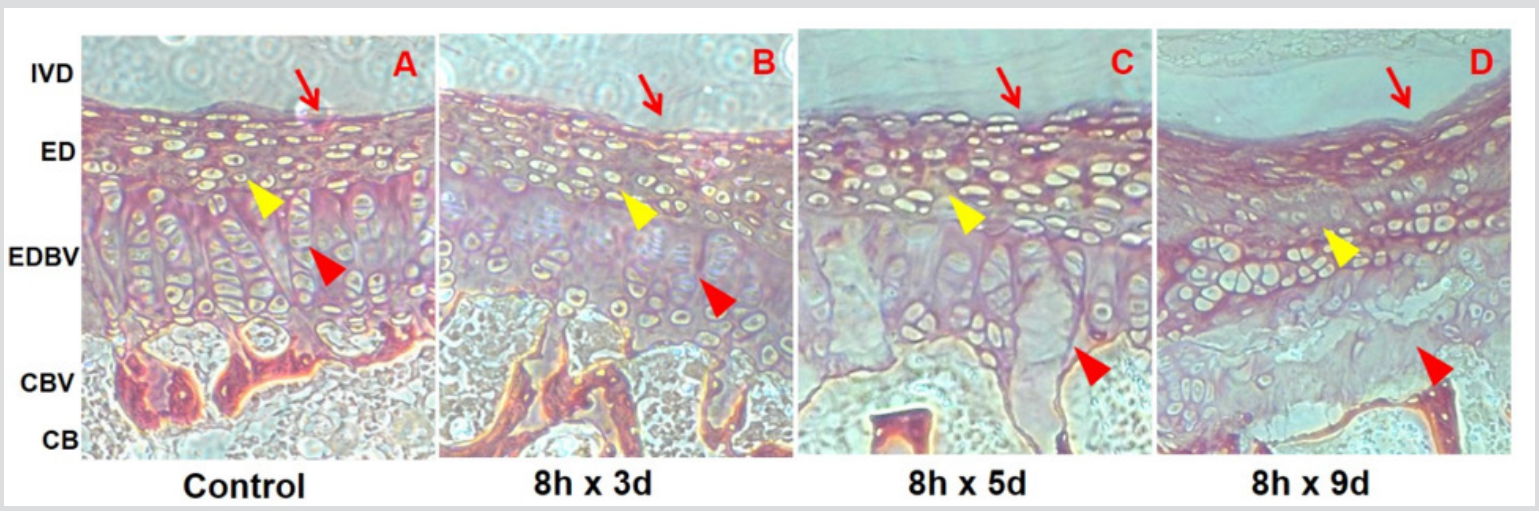

Figure 3: Longitude histopathology maps of mice intervertebral discs (IVD) of the lumbar fourth and fifth (L4-5) during upright standing experiment. Map A was a histopathology section of the L4-5 IVD of the control group mice, showing a smooth interface of the disc and endplate (red line arrow), clear and well-arranged cartilage cellular and interstitial structures (yellow triangle arrow) and intact endplate vascularity-like nutrition channels (red triangle arrow). Map B showed the channels swollen (red triangle arrow) but the endplate cells (yellow triangle arrow) and the interface (red line arrow) no evident changes. Map $\mathrm{C}$ displayed the channels structures disruption (red triangle arrow), the endplate cells and intercellular spaces edema (yellow triangle arrow) and the interface mildly collapsion and blurring. Map D showed the channels disintegration (red triangle arrow), the cells lysis and sparse distribution (yellow triangle arrow) and the endplate collapsion (red line arrow).

\section{Discussion}

\section{Dynamic Changes of ROS and SOD in IVDD Modeling}

Overload-induced-IVDD is suggested to have a multifactorial pathogenesis, in which oxidative stress plays an important role in the metabolic disorders in both bone and marrow [13]. In the present study, we examined the expression of two respective oxidative stress indicators, namely ROS and SOD. From the pre-test, we identified that both ROS and SOD are critical indicators for the development of oxidative stress in the centrums $[14,15]$. To better display the ROS and SOD distribution during the IVDD progress, we designed a histopathology and MRI dynamic monitoring program through multiple time points. Immunohistochemistry reflects biochemical changes of the IVDD both qualitatively and quantitatively. It was found 
that the ROS and SOD had a reversed change of growth and decline. The anti-oxidative stress of SOD boosted after upstanding experiment and reached the peak at day 3, and then declined gradually, but ROS activity just went up. Histopathology demonstrated that the endplate cells' inflammatory response happened at the time, showing the endplate vascularity-like nutrition channel swollen, then developing to channels disruption, edema of the endplate cartilage cells and intercellular spaces and mild collapse and blurring at the interface on day 5. Finally, by day 9, we observed channels disintegration, the cells lysis and sparse distribution, and the endplate collapse.

The OS levels increased with overloading time. The endogenous excessive ROS production, antioxidant defense homeostasis or impaired antioxidant defense mechanisms during inflammation response caused by overload-induced oxidative stress on the vertebrae determine the degree of IVDD. The tissue damage indicators changed as the overall morphology changed. At day 1 to 3 of the early stage, the antioxidant defense of SOD plays a prominent role in holdingup the activity of ROS; however, with the mechanic stress of upstanding overload continuing, the SOD was exhausted, and then the ROS production boosted a "rush" inflammatory response of the endplate, leading to endplate cells apoptosis, disruption and further IVD injury. Thus, we concluded that the SOD exhaustion and ROS activation promote IVDD launch and progress.

Limitation of the study and further exploring. We have combined a histopathological immunohistochemical technique to disclose the interrelations of OS indexes during the initiation of IVDD. The overlabour-induced IVDD animal model provides us with a possible platform to investigate the mechanism of IVDD in the ultra-early stage. However, there are some obstacles: firstly, during the animal observation phase, it was necessary to euthanize some mice for histology and molecular biology tests, that is, the animals had to be sacrificed as time progressed. Second, it is true that the tissue structure changes could be demonstrated by histological staining, but some other specialized techniques may be superior to reveal the microstructure changes in the present model. Consequently, conclusive results on the trigger mechanism of IVDD require further studies.

\section{Conclusion}

Wecombinedhistopathologicalandimmunohistochemical methods to potentially provide timely information on Oxidative stress of ROS and antioxidant defense of SOD. It was determined that OS plays a key role in mechanical stressinduced inflammation-related IVDD in mice, an increase of oxidative stress of ROS and a decrease of antioxidant defense of SOD triggering the IVDD. In future studies, we will focus on the time arrangement of the IVDD model, such as increasing the time or appropriately improving the strength of the mice on standing. Therefore, we may be able to accelerate and observe any dynamic cellular damages and draw further conclusions on the actual mechanism of the cellular damage. These findings would potentially be benefit human by allowing selection of anti-oxidative and anti-inflammation foods for halting disc degeneration.

\section{Acknowledgement}

Thank Zijing Li Ph.D. for her guidance of the experiment design and manuscript drafting.

Thank Qiang Wang M.M. for his help of animals feeding and histopathologic specimens harvest.

Thank Guoying Shen M.S. for his help of the MRI of IVDD models.

Thank the Center of Molecular Imaging and Translational Medicine of Xiamen University for the team offering the experiment platform and facilities support.

\section{Funding}

fund(2019Y3007)

\section{References}

1. Alvarez-Garcia O, Matsuzaki T, Olmer M, Masuda K, Lotz MK (2017) Agerelated reduction in the expression of FOXO transcription factors and correlations with intervertebral disc degeneration. J Orthop Res 35(12): 2682-2691.

2. Nasto LA, Robinson AR, Ngo K, Clauson CL, Dong Q et al. (2013) Mitochondrial-derived reactive oxygen species (ROS) play a causal role in aging-related intervertebral disc degeneration. J Orthop Res 31(7): 1150-1157.

3. Zorov DB, Juhaszova M, Sollott SJ (2014) Mitochondrial Reactive Oxygen Species (ROS) and ROS-induced ROS release. Physiol Rev 94(3): 909950.

4. Risbud MV, Shapiro IM (2014) Role of cytokines in intervertebral disc degeneration: Pain and disc content. Nat Rev Rheumatol 10(1): 44-56.

5. Fiorentino TV, Prioletta A, Zuo P, Folli F (2013) Hyperglycemia-induced oxidative stress and its role in diabetes mellitus related cardiovascular diseases. Curr Pharm Des 19(32): 5695-5703.

6. Yuan W, Che W, Jiang YQ, Yuan FL, Wang HR, et al. (2015) Establishment of intervertebral disc degeneration model induced by ischemic subendplate in rat tail. Spine J 15(5): 1050-1059.

7. Maria Molinos, Catarina R Almeida, Joana Caldeira, Carla Cunha, Raquel M Gonçalves, et al. (2015) Inflammation in intervertebral disc degeneration and regeneration. J R Soc Interface 12(104): 20141191.

8. Borut Poljsak, Dušan Šuput, Irina Milisav (2013) Achieving the balance between ROS and antioxidants: when to use the synthetic antioxidants. Oxid Med Cell Longev 2013, pp. 956792.

9. Robaczewska J, Kedziora-Kornatowska K, Kozakiewicz M, ZarySikorska E, Pawluk H, et al. (2016) Role of glutathione metabolism and glutathione-related antioxidant defense systems in hypertension. J Physiol Pharmacol 67(3): 331-337.

10. Natarajan RN, Williams JR, Andersson GB (2006) Modeling changes in intervertebral disc mechanics with degeneration. J Bone Joint Surg Am 88(Suppl 2): 36-40. 
11. Xu YQ Zhang ZH, Zheng YF, Feng SQ (2016) Dysregulated miR133a mediates loss of type II collagen by directly targeting Matrix Metalloproteinase 9 (MMP9) in human intervertebral disc degeneration. 41(12): E717-E724.

12. Rittie L (2017) Method for picrosirius red-polarization detection of collagen fibers in tissue sections. Methods Mol Biol 1627: 395-407.

\section{ISSN: 2574-1241}

DOI: 10.26717/BJSTR.2019.16.002801

Lin Ouyang. Biomed J Sci \& Tech Res

(c) (P) This work is licensed under Creative

Submission Link: https://biomedres.us/submit-manuscript.php
13. Hou G, Lu H, Chen M, Yao H, Zhao H (2014) Oxidative stress participates in age-related changes in rat lumbar intervertebral discs. Arch Gerontol Geriatr 59(3): 665-669.

14. Suzuki S, Fujita N, Hosogane N, Watanabe K, Ishii K, et al. (2015) Excessive reactive oxygen species are therapeutic targets for intervertebral disc degeneration. Arthritis Res Ther 17: 316.

$\begin{array}{ll}\text { BIOMEDICAL } & \text { Assets of Publishing with us } \\ \text { RESEARCHES } & \text { Global archiving of articles } \\ & \text { - Immediate, unrestricted online access } \\ \end{array}$

